\title{
Lattictic non-archimedean random stability of ACQ functional equation
}

Yeol Je Cho ${ }^{1}$ and Reza Saadati $2^{*^{*}}$

\author{
* Correspondence: rsaadati@eml.cc \\ ${ }^{2}$ Department of Mathematics, \\ Science and Research Branch, \\ Islamic Azad University, Tehran, I.R. \\ Iran \\ Full list of author information is \\ available at the end of the article
}

\begin{abstract}
In this paper, we prove the generalized Hyers-Ulam stability of the following additive-cubic-quartic functional equation

$$
\begin{aligned}
& 11 f(x+2 y)+11 f(x-2 y) \\
& \quad=44 f(x+y)+44 f(x-y)+12 f(3 y)-48 f(2 y)+60 f(y)-66 f(x)
\end{aligned}
$$

in various complete lattictic random normed spaces.

Mathematics Subject Classification (2000) Primary 54E40; Secondary 39B82, 46S50, $46 S 40$.

Keywords: Stability, Random normed space, Fixed point, Generalized Hyers-Ulam stability, Additive-cubic-quartic functional equation, Lattice, non-Archimedean normed spaces
\end{abstract}

\section{Introduction}

Probability theory is a powerful hand set for modeling uncertainty and vagueness in various problems arising in the field of science and engineering. It has also very useful applications in various fields, e.g., population dynamics, chaos control, computer programming, nonlinear dynamical systems, nonlinear operators, statistical convergence and others. The random topology proves to be a very useful tool to deal with such situations where the use of classical theories breaks down. The usual uncertainty principle of Werner Heisenberg leads to a generalized uncertainty principle, which has been motivated by string theory and non-commutative geometry. In strong quantum gravity, regime space-time points are determined in a random manner. Thus, impossibility of determining the position of particles gives the space-time a random structure. Because of this random structure, position space representation of quantum mechanics breaks down and so a generalized normed space of quasi-position eigenfunction is required. Hence one needs to discuss on a new family of random norms. There are many situations where the norm of a vector is not possible to be found and the concept of random norm seems to be more suitable in such cases, i.e., we can deal with such situations by modeling the inexactness through the random norm.

The stability problem of functional equations originated from a question of Ulam [1] concerning the stability of group homomorphisms. Hyers [2] gave a first affirmative partial answer to the question of Ulam for Banach spaces. Hyers' Theorem was generalized by Aoki [3] for additive mappings and by Rassias [4] for linear mappings by

\section{Springer}

(C) 2011 Cho and Saadati; licensee Springer. This is an Open Access article distributed under the terms of the Creative Commons Attribution License (http://creativecommons.org/licenses/by/2.0), which permits unrestricted use, distribution, and reproduction in any medium, provided the original work is properly cited. 
considering an unbounded Cauchy difference. The paper of Rassias [4] has provided a lot of influence in the development of what we call generalized Hyers-Ulam stability or as Hyers-Ulam-Rassias stability of functional equations. A generalization of the Rassias theorem was obtained by Găvruta [5] by replacing the unbounded Cauchy difference by a general control function in the spirit of Rassias approach.

The stability problems of several functional equations have been extensively investigated by a number of authors and there are many interesting results concerning this problem (see [4,6-27]).

In $[28,29]$, Jun and Kim considered the following cubic functional equation

$$
f(2 x+y)+f(2 x-y)=2 f(x+y)+2 f(x-y)+12 f(x) .
$$

It is easy to show that the function $f(x)=x^{3}$ satisfies the functional equation (2), which is called a cubic functional equation and every solution of the cubic functional equation is said to be a cubic mapping.

In [8], Lee et al. considered the following quartic functional equation

$$
f(2 x+y)+f(2 x-y)=4 f(x+y)+4 f(x-y)+24 f(x)-6 f(y) .
$$

It is easy to show that the function $f(x)=x^{4}$ satisfies the functional equation (3), which is called a quartic functional equation and every solution of the quartic functional equation is said to be a quartic mapping.

Let $X$ be a set. A function $d: X \times X \rightarrow[0, \infty]$ is called a generalized metric on $X$ if $d$ satisfies the following conditions:

(1) $d(x, y)=0$ if and only if $x=y$;

(2) $d(x, y)=d(y, x)$ for all $x, y \in X$;

(3) $d(x, z) \leq d(x, y)+d(y, z)$ for all $x, y, z \in X$.

We recall a fundamental result in fixed point theory.

Theorem 1.1. [30,31]Let $(X, d)$ be a complete generalized metric space and $J: X \rightarrow X$ be a strictly contractive mapping with Lipschitz constant $L<1$. Then, for any $x \in X$, either

$$
d\left(J^{n} x, J^{n+1} x\right)=\infty
$$

for all nonnegative integers $n$ or there exists a positive integer $n_{0}$ such that

(1) $d\left(J^{n} x, J^{n+1} x\right)<\infty$ for all $n \geq n_{0}$;

(2) the sequence $\left\{J^{n} x\right\}$ converges to a fixed point $y^{*}$ of $J$;

(3) $y^{*}$ is the unique fixed point of $J$ in the set $Y=\left\{y \in X \mid d\left(J^{n_{0}} x, y\right)<\infty\right\}$;

(4) $d\left(y, \gamma^{*}\right) \leq \frac{1}{1-L} d(y$, , $y)$ for all $y \in Y$.

In 1996, Isac and Rassias [32] were the first to provide applications of stability theory of functional equations for the proof of new fixed point theorems with applications. Using fixed point methods, the stability problems of several functional equations have been extensively investigated by a number of authors (see [33-38]). 


\section{Preliminaries}

The theory of random normed spaces (RN-spaces) is important as a generalization of deterministic result of linear normed spaces and also in the study of random operator equations. The $\mathrm{RN}$-spaces may also provide us the appropriate tools to study the geometry of nuclear physics and have important application in quantum particle physics. The generalized Hyers-Ulam stability of different functional equations in random normed spaces, RN-spaces and fuzzy normed spaces has been recently studied by Alsina [39], Mirmostafaee, Mirzavaziri and Moslehian [40,35], Miheț, and Radu [41], Miheț, et al. [42,43], Baktash et. al [44], Najati [45] and Saadati et. al. [24].

Let $\mathcal{L}=\left(L, \geq_{L}\right)$ be a complete lattice, i.e., a partially ordered set in which every nonempty subset admits supremum and infimum and $0_{\mathcal{L}}=$ infL, $1_{\mathcal{L}}=$ supL. The space of latticetic random distribution functions, denoted by $\Delta_{L}^{+}$, is defined as the set of all mappings $F: \mathbb{R} \cup\{-\infty,+\infty\} \rightarrow L$ such that $F$ is left continuous, non-decreasing on $\mathbb{R}$ and $F(+\infty)=1_{\mathcal{L}}, F(+\infty)=1_{\mathcal{L}}$.

The subspace $D_{L}^{+} \subseteq \Delta_{L}^{+}$is defined as $D_{L}^{+}=\left\{F \in \Delta_{L}^{+}: l^{-} F(+\infty)=1_{\mathcal{L}}\right\}$, where $l^{-} f(x)$ denotes the left limit of the function $f$ at the point $x$. The space $\Delta_{L}^{+}$is partially ordered by the usual point-wise ordering of functions, i.e., $F \geq G$ if and only if $F(t) \geq_{L} G(t)$ for all $t$ in $\mathbb{R}$. The maximal element for $\Delta_{L}^{+}$in this order is the distribution function given by

$$
\varepsilon_{0}(t)=\left\{\begin{array}{l}
0_{\mathcal{L}}, \text { if } t \leq 0 \\
1_{\mathcal{L}}, \text { if } t>0
\end{array}\right.
$$

Definition 2.1. [46] A triangular norm (t-norm) on $L$ is a mapping $\mathcal{T}:(L)^{2} \rightarrow L$ satisfying the following conditions:

(1) $(\forall x \in L)\left(\mathcal{T}\left(x, 1_{\mathcal{L}}\right)=x\right)$ (: boundary condition);

(2) $\left(\forall(x, y) \in(L)^{2}\right)(\mathcal{T}(x, y)=\mathcal{T}(y, x))$ (: commutativity);

(3) $\left(\forall(x, y, z) \in(L)^{3}\right)(\mathcal{T}(x, \mathcal{T}(y, z))=\mathcal{T}(\mathcal{T}(x, y), z))$ (: associativity);

(4) $\left(\forall\left(x, x^{\prime}, y, y^{\prime}\right) \in(L)^{4}\right)\left(x \leq_{L} x^{\prime}\right.$ and $\left.y \leq_{L} y^{\prime} \Rightarrow \mathcal{T}(x, y) \leq_{L} \mathcal{T}\left(x^{\prime}, y^{\prime}\right)\right)$ (: monotonicity).

Let $\left\{x_{n}\right\}$ be a sequence in $L$ converges to $x \in L$ (equipped the order topology). The $t$ norm $\mathcal{T}$ is called a continuous t-norm if

$$
\lim _{n \rightarrow \infty} \mathcal{T}\left(x_{n}, y\right)=\mathcal{T}(x, y)
$$

for any $y \in L$.

A $t$-norm $\mathcal{T}$ can be extended (by associativity) in a unique way to an $n$-array operation taking for $\left(x_{1}, \ldots, x_{n}\right) \in L^{n}$ the value $\mathcal{T}\left(x_{1}, \ldots, x_{n}\right)$ defined by

$$
\mathcal{T}_{i=1}^{0} x_{i}=1, \quad \mathcal{T}_{i=1}^{n} x_{i}=\mathcal{T}\left(\mathcal{T}_{i=1}^{n-1} x_{i}, x_{n}\right)=\mathcal{T}\left(x_{1}, \ldots, x_{n}\right) .
$$

The $t$-norm $\mathcal{T}$ can also be extended to a countable operation taking, for any sequence $\left\{x_{n}\right\}$ in $L$, the value

$$
\mathcal{T}_{i=1}^{\infty} x_{i}=\lim _{n \rightarrow \infty} \mathcal{T}_{i=1}^{n} x_{i} .
$$

The limit on the right side of (4) exists since the sequence $\left(\mathcal{T}_{i=1}^{n} x_{i}\right)_{n \in \mathbb{N}}$ is non-increasing and bounded from below. 
Note that we put $\mathcal{T}=T$ whenever $L=[0,1]$. If $T$ is a $t$-norm then, for all $x \in[0,1]$ and $n \in N \cup\{0\}, x_{T}^{(n)}$ is defined by 1 if $n=0$ and $T\left(x_{T}^{(n-1)}, x\right)$ if $n \geq 1$. A $t$-norm $T$ is said to be of Hadžić-type (we denote by $T \in \mathcal{H}$ ) if the family $\left(x_{T}^{(n)}\right)_{n \in N}$ is equicontinuous at $x=1$ (see [47]).

Definition 2.2. [46] A continuous $t$-norm $\mathcal{T}$ on $L=[0,1]^{2}$ is said to be continuous $t$-representable if there exist a continuous $t$-norm * and a continuous $t$-conorm $\diamond$ on $[0,1]$ such that, for all $x=\left(x_{1}, x_{2}\right), y=\left(y_{1}, y_{2}\right) \in L$,

$$
\mathcal{T}(x, y)=\left(x_{1} * y_{1}, x_{2} \diamond y_{2}\right) .
$$

For example,

$$
\mathcal{T}(a, b)=\left(a_{1} b_{1}, \min \left\{a_{2}+b_{2}, 1\right\}\right)
$$

and

$$
\mathbf{M}(a, b)=\left(\min \left\{a_{1}, b_{1}\right\}, \max \left\{a_{2}, b_{2}\right\}\right)
$$

for all $a=\left(a_{1}, a_{2}\right), b=\left(b_{1}, b_{2}\right) \in[0,1]^{2}$ are continuous $t$-representable.

Define the mapping $\mathcal{T}_{\wedge}$ from $L^{2}$ to $L$ by

$$
\mathcal{T}_{\wedge}(x, y)=\left\{\begin{array}{l}
x, \text { if } y \geq_{L} x \\
y, \text { if } x \geq_{L} y
\end{array}\right.
$$

Recall (see $[47,48])$ that, if $\left\{x_{n}\right\}$ is a given sequence in $L$, then $\left(\mathcal{T}_{\wedge}\right)_{i=1}^{n} x_{i}$ is defined recurrently by $\left(\mathcal{T}_{\wedge}\right)_{i=1}^{1} x_{i}=x_{1}$ and $\left(\mathcal{T}_{\wedge}\right)_{i=1}^{n} x_{i}=\mathcal{T}_{\wedge}\left(\left(\mathcal{T}_{\wedge}\right)_{i=1}^{n-1} x_{i}, x_{n}\right)$ for all $n \geq 2$.

A negation on $\mathcal{L}$ is any decreasing mapping $\mathcal{N}: L \rightarrow L$ satisfying $\mathcal{N}\left(0_{\mathcal{L}}\right)=1_{\mathcal{L}}$ and $\mathcal{N}\left(1_{\mathcal{L}}\right)=0_{\mathcal{L}}$. If $\mathcal{N}(\mathcal{N}(x))=x$ for all $x \in L$, then $\mathcal{N}$ is called an involutive negation. In the following, $\mathcal{L}$ is endowed with a (fixed) negation $\mathcal{N}$.

Definition 2.3. A latticetic random normed space is a triple $\left(X, \mu, \mathcal{T}_{\wedge}\right)$, where $X$ is a vector space and $\mu$ is a mapping from $X$ into $D_{L}^{+}$satisfying the following conditions:

$$
\begin{aligned}
& \text { (LRN1) } \mu_{x}(t)=\varepsilon_{0}(t) \text { for all } t>0 \text { if and only if } x=0 \\
& \text { (LRN2) } \mu_{\alpha x}(t)=\mu_{x}\left(\frac{t}{|\alpha|}\right) \text { for all } x \text { in } X, \alpha \neq 0 \text { and } t \geq 0 \\
& \text { (LRN3) } \mu_{x+y}(t+s) \geq_{L} \mathcal{T}_{\wedge}\left(\mu_{x}(t), \mu_{\gamma}(s)\right) \text { for all } x, y \in X \text { and } t, s \geq 0
\end{aligned}
$$

We note that, from (LPN2), it follows $\mu_{-x}(t)=\mu_{x}(t)$ for all $x \in X$ and $t \geq 0$.

Example 2.4. Let $L=[0,1] \times[0,1]$ and an operation $\leq_{L}$ be defined by

$$
\begin{gathered}
L=\left\{\left(a_{1}, a_{2}\right):\left(a_{1}, a_{2}\right) \in[0,1] \times[0,1] \text { and } a_{1}+a_{2} \leq 1\right\}, \\
\left(a_{1}, a_{2}\right) \leq_{L}\left(b_{1}, b_{2}\right) \Leftrightarrow a_{1} \leq b_{1}, a_{2} \geq b_{2}, \quad \forall a=\left(a_{1}, a_{2}\right), b=\left(b_{1}, b_{2}\right) \in L .
\end{gathered}
$$

Then $\left(L, \leq_{L}\right)$ is a complete lattice (see [46]). In this complete lattice, we denote its units by $0_{L}=(0,1)$ and $1_{L}=(1,0)$. Let $(X,\|\cdot\|)$ be a normed space. Let $\mathcal{T}(a, b)=\left(\min \left\{a_{1}, b_{1}\right\}, \max \left\{a_{2}, b_{2}\right\}\right)$ for all $a=\left(a_{1}, a_{2}\right), b=\left(b_{1}, b_{2}\right) \in[0,1] \times[0,1]$ and $\mu$ be a mapping defined by

$$
\mu_{x}(t)=\left(\frac{t}{t+\|x\|}, \frac{\|x\|}{t+\|x\|}\right), \quad \forall t \in \mathbb{R}^{+} .
$$

Then, $(X, \mu, \mathcal{T})$ is a latticetic random normed spaces. 
If $\left(X, \mu, \mathcal{T}_{\wedge}\right)$ is a latticetic random normed space, then we have

$$
\mathcal{V}=\left\{V(\varepsilon, \lambda): \varepsilon>_{L} 0_{\mathcal{L}}, \lambda \in L \backslash\left\{0_{\mathcal{L}}, 1_{\mathcal{L}}\right\}\right.
$$

is a complete system of neighborhoods of null vector for a linear topology on $X$ generated by the norm $F$, where

$$
V(\varepsilon, \lambda)=\left\{x \in X: F_{x}(\varepsilon)>{ }_{L} \mathcal{N}(\lambda)\right\} .
$$

Definition 2.5. Let $\left(X, \mu, \mathcal{T}_{\wedge}\right)$ be a latticetic random normed spaces.

(1) A sequence $\left\{x_{n}\right\}$ in $X$ is said to be convergent to a point $x \in X$ if, for any $t>0$ and $\varepsilon \in L \backslash\left\{0_{\mathcal{L}}\right\}$, there exists a positive integer $N$ such that $\mu_{x_{n}-x}(t)>_{L} \mathcal{N}(\varepsilon)$ for all $n \geq$ $N$.

(2) A sequence $\left\{x_{n}\right\}$ in $X$ is called a Cauchy sequence if, for any $t>0$ and $\varepsilon \in L \backslash\left\{0_{\mathcal{L}}\right\}$, there exists a positive integer $N$ such that $\mu_{x_{n}-x_{m}}(t)>_{L} \mathcal{N}(\varepsilon)$ for all $n \geq m \geq N$.

(3) A latticetic random normed space $\left(X, \mu, \mathcal{T}_{\wedge}\right)$ is said to be complete if every Cauchy sequence in $\mathrm{X}$ is convergent to a point in $X$.

Theorem 2.6. If $\left(X, \mu, \mathcal{T}_{\wedge}\right)$ is a latticetic random normed space and $\left\{x_{n}\right\}$ is a sequence such that $x_{n} \rightarrow x$, then $\lim _{n \rightarrow \infty} \mu_{x_{n}}(t)=\mu_{x}(t)$.

Proof. The proof is the same as classical random normed spaces (see [49]). $\square$

Lemma 2.7. Let $\left(X, \mu, \mathcal{T}_{\wedge}\right)$ be a latticetic random normed space and $x \in X$. If

$$
\mu_{x}(t)=C, \quad \forall t>0,
$$

then $C=1_{\mathcal{L}}$ and $x=0$.

Proof. Let $\mu_{x}(t)=C$ for all $t>0$. Since $\operatorname{Ran}(\mu) \subseteq D_{L}^{+}$, we have $C=1_{\mathcal{L}}$ and, by (LRN1), we conclude that $x=0$.

\section{Non-Archimedean Lattictic random normed space}

By a non-Archimedean field, we mean a field $\mathcal{K}$ equipped with a function (valuation) $\mid$. | from $K$ into $[0, \infty)$ such that $|r|=0$ if and only if $r=0,|r s|=|r||s|$ and $|r+s| \leq$ $\max \{|r|,|s|\}$ for all $r, s \in \mathcal{K}$. Clearly, $|1|=|-1|=1$ and $|n| \leq 1$ for all $n \in \mathbb{N}$. By the trivial valuation we mean the mapping $|\cdot|$ taking everything but 0 into 1 and $|0|=0$.

Let $\mathcal{X}$ be a vector space over a field $\mathcal{K}$ with a non-Archimedean non-trivial valuation $|\cdot|$. A function $\|\cdot\|: \mathcal{X} \rightarrow[0, \infty)$ is called a non-Archimedean norm, if it satisfies the following conditions:

(1) $\|x\|=0$ if and only if $x=0$;

(2) for any $r \in \mathcal{K}, x \in \mathcal{X},\|r x\|=|r| \| x||$;

(3) the strong triangle inequality (ultrametric), i.e.,

$$
\|x+y\| \leq \max \{\|x\|,\|y\|\}, \quad \forall x, y \in \mathcal{X}) .
$$

Then $(\mathcal{X},\|\cdot\|)$ is called a non-Archimedean normed space.

Due to the fact that

$$
\left\|x_{n}-x_{m}\right\| \leq \max \left\{|| x_{j+1}-x_{j} \|: m \leq j \leq n-1\right\}, \quad \forall m, n \in \mathbb{N}(n>m),
$$

a sequence $\left\{x_{n}\right\}$ is a Cauchy sequence if and only if $\left\{x_{n+1}-x_{n}\right\}$ converges to zero in a non-Archimedean normed space. By a complete non-Archimedean normed space, we mean one in which every Cauchy sequence is convergent. 
In 1897, Hensel [50] discovered the $p$-adic numbers as a number theoretical analogue of power series in complex analysis. Fix a prime number $p$. For any nonzero rational number $x$, there exists a unique integer $n_{x} \in \mathbb{Z}$ such that $x=\frac{a}{b} p^{n_{x}}$, where $a$ and $b$ are integers not divisible by $p$. Then, $|x|_{p}:=p^{-n_{x}}$ defines a non-Archimedean norm on $\mathbb{Q}$. The completion of $\mathbb{Q}$ with respect to the metric $d(x, y)=|x-y|_{p}$ is denoted by $\mathbb{Q}_{p}$, which is called the $p$-adic number field.

Throughout the paper, we assume that $\mathcal{X}$ is a vector space and $\mathcal{Y}$ is a complete nonArchimedean normed space.

Definition 3.1. A non-Archimedean lattictic random normed space (briefly, nonArchimedean LRN-space) is a triple $(\mathcal{X}, \mu, \mathcal{T})$, where $X$ is a linear space over a nonArchimedean field $\mathcal{K}, \mathcal{T}$ is a continuous $t$-norm and is $\mu$ is a mapping from $\mathcal{X}$ into $D_{L}^{+}$ satisfying the following conditions hold:

(NA-LRN1) $\mu_{x}(t)=\varepsilon_{0}(t)$ for all $t>0$ if and only if $x=0$;

(NA-LRN2) $\mu_{\alpha x}(t)=\mu_{x}\left(\frac{t}{|\alpha|}\right)$ for all $x \in \mathcal{X}, t>0, \alpha \neq 0$;

(NA-LRN3) $\mu_{x+y}(\max \{t, s\}) \geq{ }_{L} \mathcal{T}\left(\mu_{x}(t), \mu_{y}(s)\right)$ for all $x, y, z \in \mathcal{X}$ and $t, s \geq 0$.

It is easy to see that, if (NA-LRN3) holds, then we have

(RN3) $\mu_{x+y}(t+s) \geq_{L} \mathcal{T}\left(\mu_{x}(t), \mu_{y}(s)\right)$.

As a classical example, if $(\mathcal{X},\|\|$.$) is a non-Archimedean normed linear space, then$ the triple $(\mathcal{X}, \mu, \mathcal{T})$, where $L=[0,1], \mathcal{T}=\min$ and

$$
\mu_{x}(t)= \begin{cases}0, & t \leq\|x\| \\ 1, & t>\|x\|\end{cases}
$$

is a non-Archimedean LRN-space.

Example 3.2. Let $(\mathcal{X},\|\|$.$) be is a non-Archimedean normed linear space in which L$ $=[0,1]$. Define

$$
\mu_{x}(t)=\frac{t}{t+\|x\|}, \quad \forall x \in \mathcal{X}, t>0 .
$$

Then $(\mathcal{X}, \mu, \min )$ is a non-Archimedean RN-space.

Definition 3.3. Let $(\mathcal{X}, \mu, \mathcal{T})$ be a non-Archimedean LRN-space and $\left\{x_{n}\right\}$ be a sequence in $\mathcal{X}$.

(1) The sequence $\left\{x_{n}\right\}$ is said to be convergent if there exists $x \in \mathcal{X}$ such that

$$
\lim _{n \rightarrow \infty} \mu_{x_{n}-x}(t)=1_{\mathcal{L}}
$$

for all $t>0$. In that case, $x$ is called the limit of the sequence $\left\{x_{n}\right\}$.

(2) The sequence $\left\{x_{n}\right\}$ in $\mathcal{X}$ is called a Cauchy sequence if, for any $\varepsilon \in L \backslash\left\{0_{\mathcal{L}}\right\}$ and $t>$ 0 , there exists a poisitve integer $n_{0}$ such that, for all $n \geq n_{0}$ and $p>0$, $\mu_{x_{n+p}-x_{n}}(t)>_{L} \mathcal{N}(\varepsilon)$.

(3) If every Cauchy sequence is convergent, then the random norm is said to be complete and the non-Archimedean $\mathrm{RN}$-space is called a non-Archimedean random Banach space. 
Remark 3.4. [51] Let $\left(\mathcal{X}, \mu, \mathcal{T}_{\wedge}\right)$ be a non-Archimedean LRN-space. Then, we have

$$
\mu_{x_{n+p}-x_{n}}(t) \geq_{L} \mathcal{T}_{\wedge}\left\{\mu_{x_{n+j+1}-x_{n+j}}(t): j=0,1,2, \ldots, p-1\right\} .
$$

Thus the sequence $\left\{x_{n}\right\}$ is Cauchy sequence if, for any $\varepsilon \in L \backslash\left\{0_{\mathcal{L}}\right\}$ and $t>0$, there exists a positive integer $n_{0}$ such that, for all $n \geq n_{0}$,

$$
\mu_{x_{n+1}-x_{n}}(t)>_{L} \mathcal{N}(\varepsilon) .
$$

\section{Generalized Ulam-Hyers stability for functional equation (1): an odd case in non-Archimedean LRN-spaces}

Let $\mathcal{K}$ be a non-Archimedean field, $\mathcal{X}$ be a vector space over $\mathcal{K}$ and $(\mathcal{Y}, \mu, T)$ be a nonArchimedean random Banach space over $\mathcal{K}$ In this section, we investigate the stability of the functional equation (1): an odd case where $f$ is a mapping from $\mathcal{K}$ to $\mathcal{Y}$.

Let $\Psi$ be a distribution function on $\mathcal{X} \times \mathcal{X}$ to $D_{L}^{+}\left(\Psi(x, y, t)\right.$ denoted by $\Psi_{x, y}(t)$ such that

$$
\Psi_{c x, c x}(t) \geq_{L} \Psi_{x, x}\left(\frac{t}{|c|}\right), \quad \forall x \in \mathcal{X}, c \neq 0 .
$$

Definition 4.1. A mapping $f: \mathcal{X} \rightarrow \mathcal{Y}$ is said to be $\Psi$-approximately mixed ACQ if

$$
\mu_{D f(x, y)}(t) \geq_{L} \Psi_{x, y}(t), \quad \forall x, y \in \mathcal{X}, t>0 .
$$

We assume that $2 \neq 0$ in $\mathcal{K}$ (i.e., the characteristic of $\mathcal{K}$ is not 2). Our main result, in this section, is as follows:

Theorem 4.2. Let $\mathcal{K} b$ e a non-Archimedean field, X be a vector space over $\mathcal{K}$ and $(\mathcal{Y}, \mu, T)$ be a non-Archimedean complete LRN-space over $\mathcal{K} L$ et $f: \mathcal{X} \rightarrow \mathcal{Y}$ be an odd and $\Psi$-approximately mixed ACQ mapping. If, for some $\alpha \in \mathbb{R}, \alpha>0$, and some integer $k, k>3$ with $\left|2^{k}\right|<\alpha$,

$$
\Psi_{2^{-k} x, 2^{-k} y}(t) \geq \Psi_{x, y}(\alpha t), \quad \forall x \in \mathcal{X}, t>0,
$$

and

$$
\lim _{n \rightarrow \infty} \mathrm{T}_{j=n}^{\infty} M\left(x, \frac{\alpha^{j} t}{|2|^{k j}}\right)=1_{\mathcal{L}}, \quad \forall x \in \mathcal{X}, t>0,
$$

then there exists a unique cubic mapping $C: \mathcal{X} \rightarrow \mathcal{Y}$ such that

$$
\mu_{f(x)-C(x)}(t) \geq_{L} \mathcal{T}_{i=1}^{\infty} M\left(x, \frac{\alpha^{i+1} t}{|2|^{k i}}\right), \quad \forall x \in \mathcal{X}, t>0,
$$

where

$$
M(x, t):=T\left(\Psi_{x, 0}(t), \Psi_{2 x, 0}(t), \ldots, \Psi_{2^{k-1} x, 0}(t)\right), \quad \forall x \in \mathcal{X}, t>0 .
$$

Proof. First, by induction on $j$, we show that for any $x \in \mathcal{X}, t>0$ and $j \geq 2$,

$$
\mu_{f\left(4^{j} x\right)-25 \sigma^{j} f(x)}(t) \geq M_{j}(x, t):=T\left(\Psi(x, 0, t), \ldots, \Psi\left(4^{j-1} x, 0, t\right)\right) .
$$

Putting $y=0$ in (5), we obtain

$$
\mu_{f(4 x)-256 f(x)}(t) \geq \Psi(x, 0, t), \quad \forall x \in \mathcal{X}, t>0 .
$$


This proves (9) for $j=2$. Assume that (9) holds for some $j \geq 2$. Replacing $y$ by 0 and $x$ by $4^{j} x$ in (5), we get

$$
\mu_{f\left(4^{j+1} x\right)-256 f\left(4^{j} x\right)}(t) \geq \Psi\left(4^{j} x, 0, t\right), \quad \forall x \in \mathcal{X}, t>0 .
$$

Since $|256| \leq 1$, we have

$$
\begin{aligned}
\mu_{f\left(4^{j+1} x\right)-256^{j+1} f(x)}(t) & \geq T\left(\mu_{f\left(4^{j+1} x\right)-256 f\left(4^{j} x\right)}(t), \mu_{256 f\left(4^{j} x\right)-256^{j+1} f(x)}(t)\right) \\
& =T\left(\mu_{f\left(4^{j+1} x\right)-256 f\left(4^{j} x\right)}(t), \mu_{f\left(4^{j} x\right)-25 \sigma^{j} f(x)}\left(\frac{t}{|256|}\right)\right) \\
& \geq T\left(\mu_{f\left(4^{j+1} x\right)-256 f\left(4^{j} x\right)}(t), \mu_{f\left(4^{j} x\right)-25 \sigma^{j} f(x)}(t)\right) \\
& \geq T\left(\Psi\left(4^{j} x, 0, t\right), M_{j}(x, t)\right) \\
& =M_{j+1}(x, t), \quad \forall x \in \mathcal{X} .
\end{aligned}
$$

Thus (9) holds for all $j \geq 2$. In particular,

$$
\mu_{f\left(4^{k} x\right)-256^{k} f(x)}(t) \geq M(x, t), \quad \forall x \in \mathcal{X}, t>0 .
$$

Replacing $x$ by $4^{-(k n+k)} x$ in (10) and using inequality (6), we obtain

$$
\begin{aligned}
\mu_{f\left(\frac{x}{4^{k n}}\right)-256^{k} f\left(\frac{x}{4^{k n+k}}\right)}(t) & \geq M\left(\frac{x}{4^{k n+k}}, t\right) \\
& \geq M\left(x, \alpha^{n+1} t\right), \quad \forall x \in \mathcal{X}, t>0, n \geq 0 .
\end{aligned}
$$

Then, we have

$$
\mu_{\left(4^{4 k}\right)^{n} f\left(\frac{x}{\left(4^{k}\right)^{n}}\right)-\left(4^{4 k}\right)^{n+1} f\left(\frac{x}{\left(4^{k}\right)^{n+1}}\right)}(t) \geq M\left(x, \frac{\alpha^{n+1}}{\left|\left(4^{4 k}\right)^{n}\right|} t\right), \quad \forall x \in \mathcal{X}, t>0, n \geq 0,
$$

and so

$$
\begin{aligned}
& \mu_{\left(4^{4 k}\right)^{n}} f\left(\frac{x}{\left(4^{k}\right)^{n}}\right)-\left(4^{4 k}\right)^{n+p} f\left(\frac{x}{\left(4^{k}\right)^{n+p}}\right)(t) \\
& \geq T_{j=n}^{n+p}\left(\mu_{\left.\left(4^{4 k}\right)^{j} f\left(\frac{x}{\left(4^{k}\right)^{j}}\right)-\left(4^{4 k}\right)^{j+p} f\left(\frac{x}{\left(4^{k}\right)^{j+p}}\right)(t)\right)}\right. \\
& \geq T_{j=n}^{n+p} M\left(x, \frac{\alpha^{j+1}}{\mid\left(4^{4 k}\right)^{j \mid}} t\right) \\
& \geq T_{j=n}^{n+p} M\left(x, \frac{\alpha^{j+1}}{\left|\left(4^{k}\right)^{j}\right|} t\right), \quad \forall x \in \mathcal{X}, t>0, n \geq 0 .
\end{aligned}
$$

Since $\lim _{n \rightarrow \infty} \mathrm{T}_{j=n}^{\infty} M\left(x, \frac{\alpha^{j+1}}{\left|\left(4^{k}\right)^{j}\right|} t\right)=1$ for all $x \in \mathcal{X}$ and $t>0,\left\{\left(4^{4 k}\right)^{n} f\left(\frac{x}{\left(4^{k}\right)^{n}}\right)\right\}$ is a Cauchy sequence in the non-Archimedean random Banach space $(\mathcal{Y}, \mu, T)$. Hence we can define a mapping $Q: \mathcal{X} \rightarrow \mathcal{Y}$ such that

$$
\lim _{n \rightarrow \infty} \mu_{\left(4^{4 k}\right)^{n} f\left(\frac{x}{\left(4^{k}\right)^{n}}\right)-Q(x)}(t)=1, \quad \forall x \in \mathcal{X}, t>0 .
$$


Next, for all $n \geq 1, x \in \mathcal{X}$ and $t>0$, we have

$$
\begin{aligned}
\mu_{f(x)-\left(4^{4 k}\right)^{n} f\left(\frac{x}{\left(4^{k}\right)^{n}}\right)}(t) & =\mu_{\sum_{i=0}^{n-1}\left(4^{4 k}\right)^{i} f\left(\frac{x}{\left(4^{k}\right)^{i}}\right)-\left(4^{4 k}\right)^{i+1} f\left(\frac{x}{\left(4^{k}\right)^{i+1}}\right)}(t) \\
& \geq \mathrm{T}_{i=0}^{n-1}\left(\mu{ }_{\left(4^{4 k}\right)^{i} f\left(\frac{x}{\left(4^{k}\right)^{i}}\right)-\left(4^{4 k}\right)^{i+1} f\left(\frac{x}{\left(4^{k}\right)^{i+1}}\right)}(t)\right) \\
& \geq \mathrm{T}_{i=0}^{n-1} M\left(x, \frac{\alpha^{i+1} t}{\left|4^{4 k}\right|^{i}}\right) .
\end{aligned}
$$

Therefore, it follows that

$$
\begin{aligned}
\mu_{f(x)-Q(x)}(t) & \geq T\left(\mu_{f(x)-\left(4^{4 k}\right)^{n} f\left(\frac{x}{\left(4^{k}\right)^{n}}\right)}(t), \mu_{\left(4^{4 k}\right)^{n} f\left(\frac{x}{\left(4^{k}\right)^{n}}\right)-Q(x)}(t)\right) \\
& \geq T\left(\mathrm{~T}_{i=0}^{n-1} M\left(x, \frac{\alpha^{i+1} t}{\left|4^{4 k}\right|^{i}}\right), \mu_{\left(4^{4 k}\right)^{n} f\left(\frac{x}{\left(4^{k}\right)^{n}}\right)-Q(x)}(t)\right) .
\end{aligned}
$$

By letting $n \rightarrow \infty$, we obtain

$$
\mu_{f(x)-Q(x)}(t) \geq \mathrm{T}_{i=1}^{\infty} M\left(x, \frac{\alpha^{i+1} t}{\left|4^{k}\right|^{i}}\right),
$$

which proves (8). Since $T$ is continuous, from a well-known result in probabilistic metric space (see [49], Chapter 12), it follows that

$$
\lim _{n \rightarrow \infty} \mu_{\Phi_{1}(x, y, k)}(t)=\mu_{\Phi_{2}(x, y)}(t), \quad \forall x, y \in \mathcal{X}, t>0,
$$

for almost all $t>0$, where

$$
\begin{aligned}
\Phi_{1}(x, y, k)= & \left(4^{k}\right)^{n} \cdot 16 f\left(4^{-k n}(x+4 y)\right)+\left(4^{k}\right)^{n} f\left(4^{-k n}(4 x-y)\right) \\
& -306\left[\left(4^{k}\right)^{n} \cdot 9 f\left(4^{-k n}\left(x+\frac{y}{3}\right)\right)+\left(4^{k}\right)^{n} f\left(4^{-k n}(x+2 y)\right)\right] \\
& -136\left(4^{k}\right)^{n} f\left(4^{-k n}(x-y)\right)+1394\left(4^{k}\right)^{n} f\left(4^{-k n}(x+y)\right) \\
& -425\left(4^{k}\right)^{n} f\left(4^{-k n} y\right)+1530\left(4^{k}\right)^{n} f\left(4^{-k n} x\right)
\end{aligned}
$$

and

$$
\begin{aligned}
\Phi_{2}(x, y)= & 16 Q(x+4 y)+Q(4 x-y)-306\left[9 Q\left(x+\frac{y}{3}\right)+Q(x+2 y)\right] \\
& -136 Q(x-y)+1394 Q(x+y)-425 Q(y)+1530 Q(x) .
\end{aligned}
$$

On the other hand, replacing $x, y$ by $4^{-k n} x, 4^{-k n} y$, respectively, in (5) and using (NARN2) and (6), we get

$$
\mu_{\Phi_{1}(x, y, k)}(t) \geq \Psi\left(4^{-k n} x, 4^{-k n} y, \frac{t}{\left|4^{k}\right|^{n}}\right) \geq \Psi\left(x, y, \frac{\alpha^{n} t}{\left|4^{k}\right|^{n}}\right), \quad \forall x, y \in \mathcal{X}, t>0 .
$$

Since $\lim _{n \rightarrow \infty} \Psi\left(x, y, \frac{\alpha^{n} t}{\left|4^{k}\right|^{n}}\right)=1$, it follows that $Q$ is a quartic mapping.

If $Q^{\prime}: \mathcal{X} \rightarrow \mathcal{Y}$ is another quartic mapping such that $\mu_{Q^{\prime}(x)-f(x)}(t) \geq M(x, t)$ for all $x \in \mathcal{X}$ and $t>0$, then, for all $n \in N, x \in \mathcal{X}$ and $t>0$, 


$$
\left.\mu_{Q(x)-Q^{\prime}(x)}(t) \geq T\left(\mu_{Q(x)-\left(4^{4 k}\right)^{n} f\left(\frac{x}{\left(4^{k}\right)^{n}}\right)}(t), \mu_{\left(4^{4 k}\right)^{n} f\left(\frac{x}{\left(4^{k}\right)^{n}}\right)-Q^{\prime}(x)}(t), t\right)\right) .
$$

Therefore, by (12), we conclude that $Q=Q$ '. This completes the proof. $\square$

Corollary 4.3. Let $\mathcal{K}$ be a non-Archimedean field, $\mathcal{X}$ be a vector space over $\mathcal{K}$ and $(\mathcal{Y}, \mu, T)$ be a non-Archimedean random Banach space over $\mathcal{K} u n d e r$ a $t$-norm $T \in \mathcal{H}$. Let $f: \mathcal{X} \rightarrow \mathcal{Y}$ be a $\Psi$-approximately quartic mapping. If, for some $\alpha \in \mathbb{R}, \alpha>0$, and some integer $k, k>3$, with $\left|4^{k}\right|<\alpha$

$$
\Psi\left(4^{-k} x, 4^{-k} y, t\right) \geq \Psi(x, y, \alpha t), \quad \forall x \in \mathcal{X}, t>0,
$$

then there exists a unique quartic mapping $Q: \mathcal{X} \rightarrow \mathcal{Y}$ such that

$$
\mu_{f(x)-Q(x)}(t) \geq \mathrm{T}_{i=1}^{\infty} M\left(x, \frac{\alpha^{i+1} t}{|4|^{k i}}\right), \quad \forall x \in \mathcal{X}, t>0,
$$

where

$$
M(x, t):=T\left(\Psi(x, 0, t), \Psi(4 x, 0, t), \ldots, \Psi\left(4^{k-1} x, 0, t\right)\right), \quad \forall x \in \mathcal{X}, t>0 .
$$

Proof. Since

$$
\lim _{n \rightarrow \infty} M\left(x, \frac{\alpha^{j} t}{|4|^{k j}}\right)=1, \quad \forall x \in \mathcal{X}, t>0,
$$

and $T$ is of Hadžić type, it follows that

$$
\lim _{n \rightarrow \infty} \mathrm{T}_{j=n}^{\infty} M\left(x, \frac{\alpha^{j} t}{|4|^{k j}}\right)=1, \quad \forall x \in \mathcal{X}, t>0 .
$$

Now, if we apply Theorem 4.2, we get the conclusion.

Example 4.4. Let $\left(\mathcal{X}, \mu, T_{M}\right)$ non-Archimedean random normed space in which

$$
\mu_{x}(t)=\frac{t}{t+\|x\|}, \quad \forall x \in \mathcal{X}, t>0,
$$

and $\left(\mathcal{Y}, \mu, T_{M}\right)$ a complete non-Archimedean random normed space (see Example 3.2). Define

$$
\Psi(x, y, t)=\frac{t}{1+t} .
$$

It is easy to see that (6) holds for $\alpha=1$. Also, since

$$
M(x, t)=\frac{t}{1+t^{\prime}}
$$

we have

$$
\begin{aligned}
\lim _{n \rightarrow \infty} \mathrm{T}_{M, j=n}^{\infty} M\left(x, \frac{\alpha^{j} t}{|4|^{k j}}\right) & =\lim _{n \rightarrow \infty}\left(\lim _{m \rightarrow \infty} \mathrm{T}_{M, j=n}^{m} M\left(x, \frac{t}{|4|^{k j}}\right)\right) \\
& =\lim _{n \rightarrow \infty} \lim _{m \rightarrow \infty}\left(\frac{t}{t+\left|4^{k}\right|^{n}}\right) \\
& =1, \quad \forall x \in \mathcal{X}, t>0 .
\end{aligned}
$$


Let $f: \mathcal{X} \rightarrow \mathcal{Y}$ be a $\Psi$-approximately quartic mapping. Thus, all the conditions of Theorem 4.2 hold and so there exists a unique quartic mapping $Q: \mathcal{X} \rightarrow \mathcal{Y}$ such that

$$
\mu_{f(x)-Q(x)}(t) \geq \frac{t}{t+\left|4^{k}\right|} .
$$

\section{Acknowledgements}

The authors would like to thank the referee and area editor Professor T. Gnana Bhaskar for giving useful suggestions and comments for the improvement of this paper. Yeol Je Cho was also supported by Basic Science Research Program through the National Research Foundation of Korea (NRF) funded by the Ministry of Education, Science and Technology (Grant Number: 2011-0021821)

\section{Author details}

${ }^{1}$ Department of Mathematics Education and Rins, Gyeongsang National University, Chinju 660-701, Korea ${ }^{2}$ Department of Mathematics, Science and Research Branch, Islamic Azad University, Tehran, I.R. Iran

\section{Authors' contributions}

All authors carried out the proof. All authors conceived of the study, and participated in its design and coordination. All authors read and approved the final manuscript.

\section{Competing interests}

The authors declare that they have no competing interests.

Received: 16 February 2011 Accepted: 5 September 2011 Published: 5 September 2011

\section{References}

1. Ulam, SM: A Collection of the Mathematical Problems. Intersci Publ New York. (1960)

2. Hyers, DH: On the stability of the linear functional equation. Proc Natl Acad Sci USA. 27, $222-224$ (1941). doi:10.1073/ pnas.27.4.222

3. Aoki, T: The stability of the linear transformation in Banach spaces. J Math Soc Japan. 2, 64-66 (1950). doi:10.2969/jmsj/ 00210064

4. Rassias, ThM: On the stability of the linear mapping in Banach spaces. Proc Am Math Soc. 72, $297-300$ (1978). doi:10.1090/S0002-9939-1978-0507327-1

5. Găvruta, P: A generalization of the Hyers-Ulam-Rassias stability of approximately additive mappings. J Math Anal Appl. 184, 431-436 (1994). doi:10.1006/jmaa.1994.1211

6. Cho, Y, Park, C, Saadati, R: Functional inequalities in non-Archimedean Banach spaces. Appl Math Lett. 23, 1238-1242 (2010). doi:10.1016/j.aml.2010.06.005

7. Hyers, DH, Isac, G, Rassias, ThM: Stability of Functional Equations in Several Variables. Birkhäuser, Basel. (1998)

8. Lee, S, Im, S, Hwang, I: Quartic functional equations. J Math Anal Appl. 307, 387-394 (2005). doi:10.1016/j. jmaa.2004.12.062

9. Park, C: Hyers-Ulam-Rassias stability of homomorphisms in quasi-Banach algebras. Bull Sci Math. 132, 87-96 (2008). doi:10.1016/j.bulsci.2006.07.004

10. Park, C: Hyers-Ulam-Rassias stability of a generalized Apollonius-Jensen type additive mapping and isomorphisms between $C^{*}$-algebras. Math Nachr. 281, 402-411 (2008). doi:10.1002/mana.200510611

11. Park, C, Cui, J: Generalized stability of $C^{*}$-ternary quadratic mappings. Abstr Appl Anal. 2007, Article ID 23282 (2007)

12. Park, C, Najati, A: Homomorphisms and derivations in $C^{*}$-algebras. Abstr Appl Anal. 2007, Article ID 80630 (2007)

13. Rassias, JM: On approximation of approximately linear mappings by linear mappings. Bull Sci Math. 108, 445-446 (1984)

14. Rassias, JM: Refined Hyers-Ulam approximation of approximately Jensen type mappings. Bull Sci Math. 131, 89-98 (2007). doi:10.1016/j.bulsci.2006.03.011

15. Rassias, JM, Rassias, MJ: Asymptotic behavior of alternative Jensen and Jensen type functional equations. Bull Sci Math. 129, 545-558 (2005). doi:10.1016/j.bulsci.2005.02.001

16. Rassias, ThM: Problem 16; 2, Report of the 27th International Symposium on Functional Equations. Aequat Math. 39 292-293 (1990)

17. Rassias, ThM: On the stability of the quadratic functional equation and its applications. Studia Univ Babes-Bolyai. XLIII, 89-124 (1998)

18. Rassias, ThM: The problem of S.M. Ulam for approximately multiplicative mappings. J Math Anal Appl. 246, 352-378 (2000). doi:10.1006/jmaa.2000.6788

19. Rassias, ThM: On the stability of functional equations in Banach spaces. J Math Anal Appl. 251, 264-284 (2000) doi:10.1006/jmaa.2000.7046

20. Rassias, ThM: On the stability of functional equations and a problem of Ulam. Acta Appl Math. 62, 23-130 (2000). doi:10.1023/A:1006499223572

21. Rassias, ThM, Šemrl, P: On the behaviour of mappings which do not satisfy Hyers-Ulam stability. Proc Am Math Soc. 114, 989-993 (1992). doi:10.1090/S0002-9939-1992-1059634-1

22. Rassias, ThM, Šemrl, P: On the Hyers-Ulam stability of linear mappings. J Math Anal Appl. 173, 325-338 (1993). doi:10.1006/jmaa.1993.1070

23. Rassias, ThM, Shibata, K: Variational problem of some quadratic functionals in complex analysis. J Math Anal Appl. 228 234-253 (1998). doi:10.1006/jmaa.1998.6129 
24. Saadati, R, Vaezpour, SM, Cho, YJ: A note on the "On the stability of cubic mappings and quadratic mappings in random normed spaces". J Inequal Appl. 2009, Article ID 214530 (2009)

25. Saadati, R, Zohdi, MM, Vaezpour, SM: Nonlinear $\mathcal{L}$-random stability of an ACQ functional equation. J Inequal Appl. 2011, Art ID 194394 (2011)

26. Saadati, R, Park, C: Non-Archimedean L-fuzzy normed spaces and stability of functional equations. Comput Math Appl. 60, 2488-2496 (2010). doi:10.1016/j.camwa.2010.08.055

27. Saadati, R, Cho, YJ, Vahidi, J: The stability of the quartic functional equation in various spaces. Comput Math Appl. 60, 1994-2002 (2010). doi:10.1016/j.camwa.2010.07.034

28. Jun, K, Kim, H: The generalized Hyers-Ulam-Rassias stability of a cubic functional equation. J Math Anal Appl. 274 867-878 (2002). doi:10.1016/50022-247X(02)00415-8

29. Jun, K, Kim, H: Hyers-Ulam-Rassias Stability of Functional Equations in Mathematical Analysis. Hadronic Press inc., Palm Harbor (2001)

30. Cădariu, L, Radu, V: Fixed points and the stability of Jensen's functional equation. J Inequal Pure Appl Math. 4, Article ID 4 (2003)

31. Diaz, J, Margolis, B: A fixed point theorem of the alternative for contractions on a generalized complete metric space. Bull Am Math Soc. 74, 305-309 (1968). doi:10.1090/S0002-9904-1968-11933-0

32. Isac, G, Rassias, ThM: Stability of $\psi$-additive mappings: Appications to nonlinear analysis. Int J Math Math Sci. 19, 219-228 (1996). doi:10.1155/S0161171296000324

33. Cădariu, L, Radu, V: On the stability of the Cauchy functional equation: a fixed point approach. Grazer Math Ber. 346, 43-52 (2004)

34. Cădariu, L, Radu, V: Fixed point methods for the generalized stability of functional equations in a single variable. Fixed Point Theory Appl. 2008, Article ID 749392 (2008)

35. Mirzavaziri, M, Moslehian, MS: A fixed point approach to stability of a quadratic equation. Bull Braz Math Soc. 37, 361-376 (2006). doi:10.1007/s00574-006-0016-z

36. Park, C: Fixed points and Hyers-Ulam-Rassias stability of Cauchy-Jensen functional equations in Banach algebras. Fixed Point Theory Appl. 2007, Article ID 50175 (2007)

37. Park, C: Generalized Hyers-Ulam-Rassias stability of quadratic functional equations: a fixed point approach. Fixed Point Theory Appl. 2008, Article ID 493751 (2008)

38. Radu, V: The fixed point alternative and the stability of functional equations. Fixed Point Theory. 4, 91-96 (2003)

39. Alsina, C: On the stability of a functional equation arising in probabilistic normed spaces. In General Inequalities, vol. 5, pp. 263-271.Oberwolfach, 1986, Birkhäuser, Basel (1987)

40. Mirmostafaee, AK, Moslehian, MS: Fuzzy approximately cubic mappings. Inf Sci. 178, 3791-3798 (2008). doi:10.1016/j. ins.2008.05.032

41. Miheț, D, Radu, V: On the stability of the additive Cauchy functional equation in random normed spaces. J Math Anal Appl. 343, 567-572 (2008)

42. Miheț, D, Saadati, R, Vaezpour, SM: The stability of the quartic functional equation in random normed spaces. Acta Appl Math. 110, 797-803 (2010). doi:10.1007/s10440-009-9476-7

43. Miheț, D, Saadati, R, Vaezpour, SM: The stability of an additive functional equation in Menger probabilistic $\varphi$-normed spaces. Math Slovaca. 61, 817-826 (2011). doi:10.2478/s12175-011-0049-7

44. Baktash, E, Cho, YJ, Jalili, M, Saadati, R, Vaezpour, SM: On the stability of cubic mappings and quadratic mappings in random normed spaces. J Inequal Appl. 2008, Article ID 902187 (2008)

45. Najati, A: Fuzzy stability of a generalized quadratic functional equation. Commun Korean Math Soc. 25(3), 405-417 (2010). doi:10.4134/CKMS.2010.25.3.405

46. Deschrijver, G, Kerre, EE: On the relationship between some extensions of fuzzy set theory. Fuzzy Sets Syst. 23, 227-235 (2003)

47. Hadžić, O, Pap, E: Fixed Point Theory in PM Spaces. Kluwer Academic Publishers, Dordrecht (2001)

48. Hadžić, $\mathrm{O}$, Pap, E, Budincević, M: Countable extension of triangular norms and their applications to the fixed point theory in probabilistic metric spaces. Kybernetica. 38, 363-381 (2002)

49. Schweizer, B, Sklar, A: Probabilistic Metric Spaces. Elsevier, New York (1983)

50. Hensel, K: Uber eine neue Begrundung der Theorie der algebraischen Zahlen. Jahres Deutsch Math Verein. 6, 83-88 (1897)

51. Mirmostafaee, M, Moslehian, MS: Fuzzy stability of additive mappings in non-Archimedean Fuzzy normed spaces. Fuzzy Sets Syst. 160, 1643-1652 (2009). doi:10.1016/j.fss.2008.10.011

doi:10.1186/1687-1847-2011-31

Cite this article as: Cho and Saadati: Lattictic non-archimedean random stability of ACQ functional equation. Advances in Difference Equations 2011 2011:31. 